\title{
How Accurate are SuperCOSMOS Positions?
}

\author{
Adam Schaefer ${ }^{1,2}$, Richard Hunstead ${ }^{1}$ and Helen Johnston ${ }^{1}$ \\ ${ }^{1}$ Sydney Institute for Astronomy, School of Physics, University of Sydney, New South Wales 2006, Australia \\ 2Email: schaefer@physics.usyd.edu.au
}

(ReCEIVED September 27, 2013; ACCEPTED January 01, 2014)

\begin{abstract}
Optical positions from the SuperCOSMOS Sky Survey have been compared in detail with accurate radio positions that define the second realisation of the International Celestial Reference Frame (ICRF2). The comparison was limited to the IIIaJ plates from the UK/AAO and Oschin (Palomar) Schmidt telescopes. A total of 1373 ICRF2 sources was used, with the sample restricted to stellar objects brighter than $B_{J}=20$ and Galactic latitudes $|b|>10^{\circ}$. Position differences showed an rms scatter of 0.16 arcsec in right ascension and declination. While overall systematic offsets were $<0.1$ arcsec in each hemisphere, both the systematics and scatter were greater in the north.
\end{abstract}

Keywords: astrometry - galaxies: active - reference systems

\section{INTRODUCTION}

The accurate measurement of optical positions for celestial objects is critical to all fields of astronomy, especially multiwavelength studies involving radio, infrared, X-ray and $\gamma$-ray observations. Traditionally, the celestial reference frame was linked to the Earth and based on the positions of Galactic stars. Stars, however, are not fixed in space and the Earth is not a stable platform. While the latter issue can be overcome with satellite observations, stellar proper motions limit the long-term accuracy of reference frames based on Galactic stars. The solution has been to adopt a quasi-inertial reference frame based on the radio positions of extragalactic objects, mostly quasars and BL Lac objects, determined using very long-baseline interferometry (VLBI).

\subsection{Astrometric reference frames}

At present the most accurate astrometric measurements at optical wavelengths are those made with the European Space Agency's (ESA) Hipparcos satellite (Perryman et al. 1997). In 1995, the Hipparcos and Tycho catalogues from this mission (mean epoch J1991.25) were tied to the official IAU reference system known as the International Celestial Reference System (ICRS). The ICRS is consistent with the FK5 (J2000) system and its axes are defined by the VLBI positions of 212 ultra-compact extragalactic sources constituting the defining sources (Ma et al. 1998) for the International Celestial Reference Frame (ICRF, now known as ICRF1); these VLBI positions have errors $<1$ mas in each coordinate.
Since the extragalactic objects were too faint for direct observation by Hipparcos, the link between the two systems was made through additional ground-based observations. The estimated uncertainty in the link is dominated by proper motion uncertainties of $\sim 0.25 \mathrm{mas} / \mathrm{yr}$.

ICRF1 was based on a catalogue of 608 extragalactic sources which served to define the ICRS from 1998 to 2009. In 2009, ICRF1 was superseded by the second realisation of the International Celestial Reference Frame, ICRF2 (Fey, Gordon, \& Jacobs 2009), which contains 295 defining sources, 97 of which were defining sources in ICRF1. Median positional accuracy for the 1217 sources (295 defining, 922 non-defining) is now $\sim 0.18$ mas in each coordinate. ICRF2 has the advantage over ICRF1 of being distributed more uniformly over the sky, although there is still a significant deficit of sources in the south. To be included in the ICRF2 defining list the sources must (ideally) show no extended structure or structure variation with time at the standard VLBI frequencies of 2.3 and $8.6 \mathrm{GHz}$. However, detailed monitoring of four ICRF2 sources with the Very Long Baseline Array (VLBA) over a year at 43, 23 and $8.6 \mathrm{GHz}$ (Fomalont et al. 2011) showed all of them to have multiple components. Although the relative positions of the identified radio cores were shown to be stationary at a level of 0.02 mas, two of the four ICRF2 sources showed detectable motion in the direction of the radio jet. Fomalont et al. (2011) conclude that the influence of moving jet components sets a fundamental limit on the present ICRF2 positions. In the optical, the upcoming ESA astrometric space mission, Gaia (Perryman et al. 2001; Mignard \& Klioner 2012), will measure positions 
for $\sim 500000$ quasars to $G=20$ with precision better than $\sim 0.2$ mas, allowing a direct link with ICRF2 at the sub-mas scale.

\subsection{The SuperCOSMOS Sky Survey (SSS)}

The Schmidt telescope surveys of the northern and southern sky form an invaluable resource for seeking optical counterparts of objects found at other wavelengths, and for transferring a coordinate reference frame onto CCD images. They represent the last all-sky photographic surveys and their digitised versions continue to serve a wide range of astronomical projects. The SuperCOSMOS Sky Survey (hereafter SSS; Hambly et al. $\left.2001 \mathrm{c}^{1}\right)$ was a high resolution $(10 \mu \mathrm{m}$ or $0.67 \mathrm{arcsec} / \mathrm{pixel}$ ) digitisation of the UK (southern) and Palomar (northern) Schmidt surveys using the SuperCOSMOS high-precision microdensitometer. Hambly et al. (1998) have demonstrated the capability of this machine to centroid wellexposed stellar images on survey-grade plates to a precision of $\sim 0.5 \mu \mathrm{m}$ or 33 mas.

The surveys covered the sky in three colours - blue (IIIaJ emulsion), red (IIIaF emulsion) and near-infrared (IVN emulsion) - with magnitudes referred to as $B_{J}, R$ and $I$, respectively. Limiting magnitudes are $\sim 22.5$ in $B_{J}, \sim 19$ in $I$ and $\sim 21$ in $R$. Plates were $6.5^{\circ}$ on a side, and taken on $5^{\circ}$ plate centres, giving substantial overlap between adjacent plates to minimise edge effects. As a result, objects near the plate boundaries were observed two or more times. In such cases the position adopted by the SSS (Hambly et al. 2001c) was taken to be the one closest to the plate centre, since position errors are known to increase with radial distance.

Positional accuracy of the SSS catalogue is claimed to be $0.1 \mathrm{arcsec}$ for objects with $B_{J}<19$. The positions themselves are tied to the Hipparcos reference frame via a second analysis of the Tycho catalogue using a more advanced reduction method (Høg et al. 2000). The Tycho-2 catalogue, in turn, is closely linked to ICRF1 (Hambly et al. 2001c).

Given the complex distortions introduced by the bending of the glass plates to the spherical focal surface of the Schmidt cameras, the goal of our investigation was to determine the positional accuracy of SuperCOSMOS over the whole sky through a detailed comparison with ICRF2. Hambly et al. (2001c) found that the smallest rms scatter between SuperCOSMOS and ICRF1 for stellar objects was for the IIIaJ plates. As a result the comparisons with ICRF2 presented in this paper have been made with SuperCOSMOS positions determined from the IIIaJ plates taken from the $1.2-\mathrm{m}$ UK/AAO Schmidt telescope at Siding Spring Observatory in Australia over the period 1974-1994, and the second epoch Palomar Observatory Sky Survey (POSS-II) plates from the 1.2-m Oschin Schmidt telescope at Palomar Observatory in California, taken over the period 1987-1999 (Hambly et al. 2001a). The UK Schmidt scans were made mostly on the original survey plates, while the POSS-II scans were made

\footnotetext{
${ }^{1}$ http://www-wfau.roe.ac.uk/sss/
}

on glass atlas copies of the survey originals. The crossover declination between the scans of the northern and southern surveys was at $+2.5^{\circ}$ (B1950).

Previous radio-optical position comparisons are presented in Section 2 and the selection criteria for the present comparison are discussed in Section 3. Results are given in Section 4, discussed in Section 5 and summarised in Section 6.

\section{PREVIOUS COMPARISONS WITH ICRF}

\subsection{USNO-A2.0}

Prior to the release of the SSS catalogue and images, the most accurate digitisation of the Schmidt plate surveys were those carried out at the US Naval Observatory (USNO) by Monet et al. (1998). The USNO-A2.0 catalogue of $>500 \times 10^{6} \mathrm{ob}-$ jects, was based on scans of POSS-I E and O plates north of declination $-18^{\circ}$ and UK Schmidt $\mathrm{J}$ and European Southern Observatory (ESO) R plates south of $-18^{\circ}$. Digitisation was at $0.9 \mathrm{arcsec} / \mathrm{pixel}$, and astrometric calibration was aligned with ICRF through the USNO ACT Reference Catalog (Urban, Corbin, \& Wycoff 1998).

A detailed comparison between USNO-A2.0 (and earlier digitised versions of the Schmidt surveys) and ICRF1 was carried out by Deutsch (1999). Of the 608 sources in ICRF1, 325 were rejected by visual inspection as being too faint, too extended to obtain a reliable centroid, or in crowded optical fields. Mean offsets for the remaining 283 objects were +0.04 arcsec in both right ascension (RA) and declination (Dec), with maximum deviations of $\approx 0.18$ arcsec in each coordinate for the best $68 \%$.

\subsection{SuperCOSMOS}

Hambly et al. (2001c) used an approach similar to that used by Deutsch (1999), with a further constraint of limiting the Galactic latitude to $|b|>30^{\circ}$. This additional constraint reduced the comparison sample to 110 stellar objects for the $\mathrm{J} / \mathrm{EJ}$ survey and somewhat fewer for the R and I surveys. The most accurate results came from the J/EJ surveys, with mean offsets of -0.02 arcsec in both RA and Dec for the 92 stellar objects with $B_{J}<20.0$ and maximum deviations of 0.11 arcsec in each coordinate for the best $68 \%$. It is important to note that the Hambly et al. (2001c) J/EJ comparison was limited to southern declinations. Given the relatively small numbers there was no attempt to explore the comparison as a function of declination.

\section{SuperCOSMOS-ICRF2 COMPARISON}

Following the results obtained by Hambly et al. (2001c), we decided to restrict our comparison with ICRF2 to SSS positions from the $\mathrm{J} / \mathrm{EJ}$ surveys. The radio positions used in the comparison come from the 1217 sources in ICRF2 (295 defining, 922 non-defining), together with a further 2197 ICRF2 compact sources comprising the VLBA Calibration 
Source (VCS) list, all with positional accuracy $<1$ mas (Fey et al. 2009).

Because of crowded star fields at low Galactic latitude, our comparison was restricted to sources with $|b|>10^{\circ}$; Hambly et al. (2001c) adopted a more stringent limit of $|b|>30^{\circ}$. A further constraint was to limit the comparison to stellar objects brighter than $B_{J}=20$, following Hambly et al. (2001c). Finally, to exclude the majority of chance associations with foreground stars, matches were sought only out to a radius of 1 arcsec. Despite this limit, spectroscopic follow-up observations (Titov et al. 2011, 2013) identified several cases where the optical field of an ICRF2 source was obscured by a closely-aligned foreground star; we believe, however, that such cases are rare and unlikely to affect the statistical results from this study.

Of the 3414 sources comprising the ICRF2, 853 were either not detected in the SSS J/EJ catalogue or were unlikely identifications with position offsets $>1$ arcsec. A further 178 sources with $|b|<10^{\circ}$ were rejected, along with 378 objects catalogued as extended and likely to be galaxies (or blends), with correspondingly larger centroiding errors. The faint magnitude limit of $B_{J}=20$, together with a bright limit of $B_{J}=15$, where image saturation can affect the positional accuracy, eliminated a further 632 sources. The final sample contained 1373 ICRF2 matches, of which 187 were from the set of 295 defining sources. The much larger size of our sample in comparison with previous studies by Deutsch (1999) and Hambly et al. (2001c) has allowed us to examine positional offsets as a function of declination.

\section{RESULTS}

Position differences between ICRF2 and SSS were calculated in arcseconds on the sky in the sense optical minus radio. This follows the convention used by Deutsch (1999) and Hambly et al. (2001c). The overall distribution of position differences for the full set of 1373 sources is shown in Figure 1. It is immediately clear from this cross-plot that there are small but significant systematic errors in the optical positions, both in RA and Dec. On the other hand, given the strong central concentration of points, it is likely that the small number of sources with radial offsets $>0.5$ arcsec are mostly chance associations.

These results are summarised in Table 1, in which position offsets and errors are given separately for the ICRF2 defining sources and for the full ICRF2 sample. The two samples are separated further into northern and southern hemispheres, with the division set at $\delta=+2.5^{\circ}$, the crossover point between the northern and southern surveys.

To investigate these errors further, and separate the contributions from the northern and southern surveys, we now examine the position differences as a function of declination in Figures 2 and 3. Group means over intervals in declination with comparable numbers of sources are shown in red.

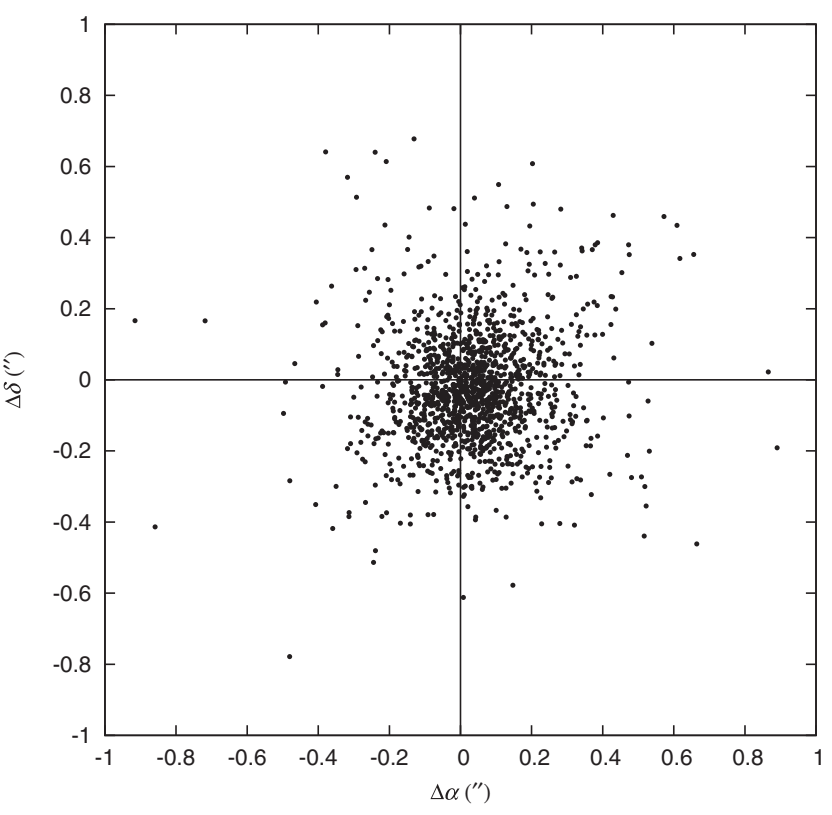

Figure 1. Plot of $\Delta \delta$ arcsec versus $\Delta \alpha$ arcsec for all 1373 ICRF2 sources in our comparison sample. Position offsets, $\Delta \alpha, \Delta \delta$, are in arcseconds in the sense optical minus radio.

\subsection{RA offset versus declination, Figure 2}

The most prominent feature of this plot is the uniform systematic offset in the north, amounting to $>10 \sigma_{\text {mean }}$, as well as a larger scatter. The offset is present in all three subsets of ICRF2: defining, non-defining and VCS. There is a smaller but significant offset in the south in the opposite sense.

\subsection{Dec offset versus declination, Figure 3}

The most striking aspect of this plot is the regular pattern of offsets in the north, with peak amplitudes as large as 0.5 arcsec, even though the mean offset is not significant. This highlights the value of our larger sample. No obvious pattern is seen in the south, although there is a significant offset of -0.05 arcsec.

\subsection{RA and Dec offsets versus magnitude}

To investigate the possibility of any systematic effects occurring as a function of apparent magnitude we have investigated the mean and scatter in RA and Dec as a function of magnitude. In both the POSS-II and UK Schmidt telescope survey regions there is no significant change in systematic offset at fainter magnitudes, although the scatter does increase for fainter objects. This is consistent with the results of Hambly et al. (2001c) and Deutsch (1999).

\section{DISCUSSION}

Hambly et al. (2001c) carried out a detailed set of comparisons between SSS and ICRF1 in the south, showing that 
Table 1. Position offsets between SSS and ICRF2, in the sense optical minus radio with $\Delta \alpha$ and $\Delta \delta$ measured in arcseconds on the sky. The defining ICRF2 sources are shown separately. Source selection is discussed in Section 3.

\begin{tabular}{|c|c|c|c|c|c|c|c|c|c|c|}
\hline & \multicolumn{5}{|c|}{ ICRF2 defining sources } & \multicolumn{5}{|c|}{ Full ICRF2 sample } \\
\hline & $N$ & $\overline{\Delta \alpha}(\operatorname{arcsec})$ & $\sigma_{\alpha}$ & $\overline{\Delta \delta}(\operatorname{arcsec})$ & $\sigma_{\delta}$ & $N$ & $\overline{\Delta \alpha}(\operatorname{arcsec})$ & $\sigma_{\alpha}$ & $\overline{\Delta \delta}(\operatorname{arcsec})$ & $\sigma_{\delta}$ \\
\hline All sources & 187 & $+0.018 \pm 0.011$ & 0.15 & $-0.013 \pm 0.012$ & 0.17 & 1373 & $+0.036 \pm 0.004$ & 0.16 & $-0.025 \pm 0.004$ & 0.16 \\
\hline North $\delta \geq+2.5^{\circ}$ & 107 & $+0.087 \pm 0.019$ & 0.18 & $+0.007 \pm 0.019$ & 0.20 & 764 & $+0.089 \pm 0.006$ & 0.17 & $-0.012 \pm 0.006$ & 0.18 \\
\hline South $\delta<+2.5^{\circ}$ & 81 & $-0.026 \pm 0.015$ & 0.13 & $-0.026 \pm 0.017$ & 0.16 & 609 & $-0.031 \pm 0.005$ & 0.13 & $-0.041 \pm 0.006$ & 0.15 \\
\hline
\end{tabular}

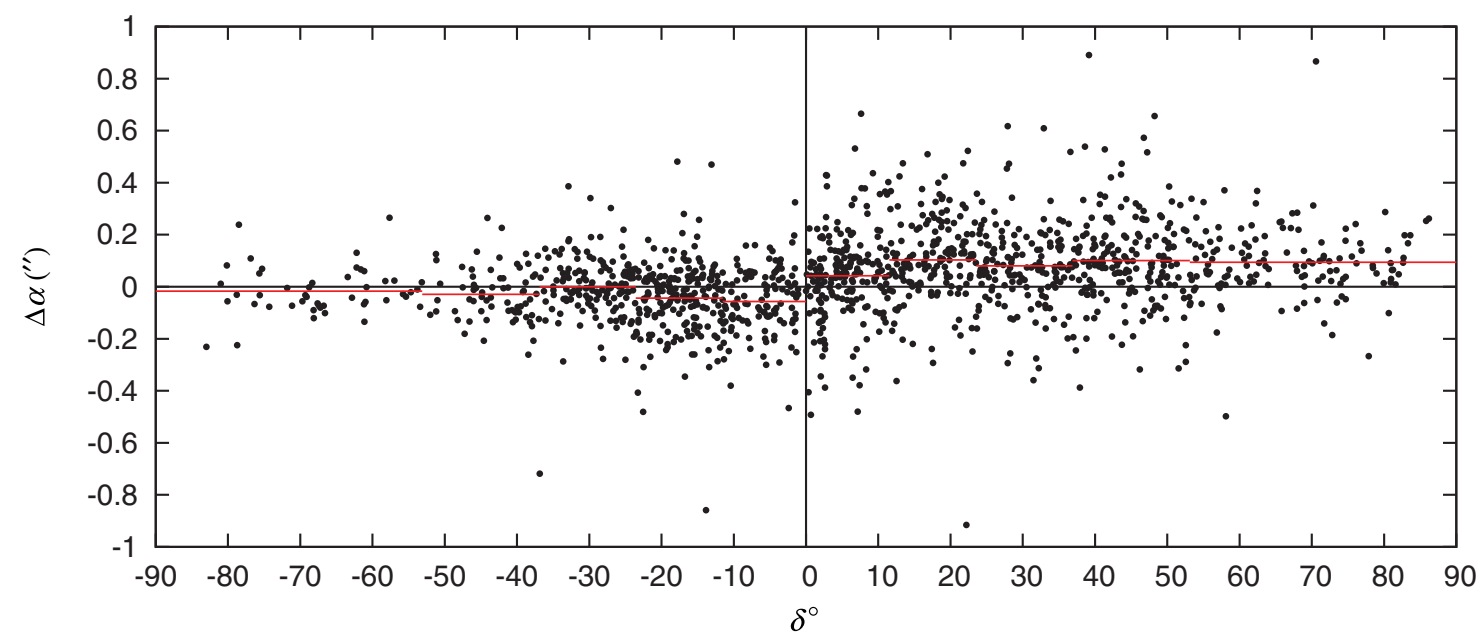

Figure 2. Plot of RA offset (arcsec on the sky, optical minus radio) versus declination (deg). The red horizontal bars are group means over intervals with comparable numbers of sources.

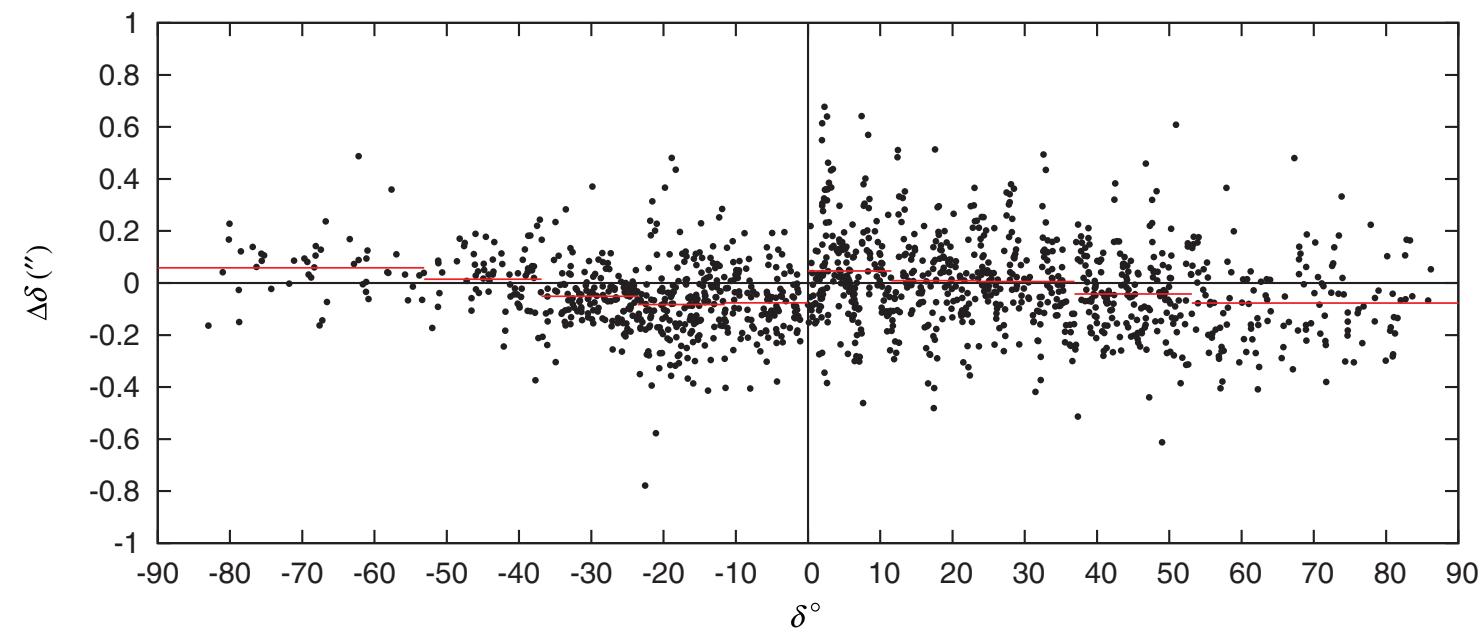

Figure 3. Plot of declination offset (arcsec, optical minus radio) versus declination (deg). The red horizontal bars are group means over intervals with comparable numbers of sources. Note the regular pattern of offsets in the north, despite a zero mean offset; this is discussed in more detail in Section 5.

zero-point errors were no larger than 100 mas for the blue and red surveys. These results are confirmed (with substantially larger numbers of sources) by the results in Table 1 . However, a similar analysis was not done for the northern POSS-II surveys, because they were not completed in time for inclusion in the definitive papers describing the SuperCOSMOS Sky Surveys (Hambly et al. 2001a, 2001c; Hambly, Irwin, and MacGillivray 2001b). In particular, the
POSS-II J survey scans were only added to the online SSS database in 2008. ${ }^{2}$

Extensive checking of the northern declination offsets confirmed that the repetitive sawtooth pattern was real. A power spectrum analysis of the offsets between declinations $+2.5^{\circ}$ and $+52.5^{\circ}$, where the effect is most prominent, is shown

\footnotetext{
${ }^{2}$ http://www-wfau.roe.ac.uk/sss/status.html
} 


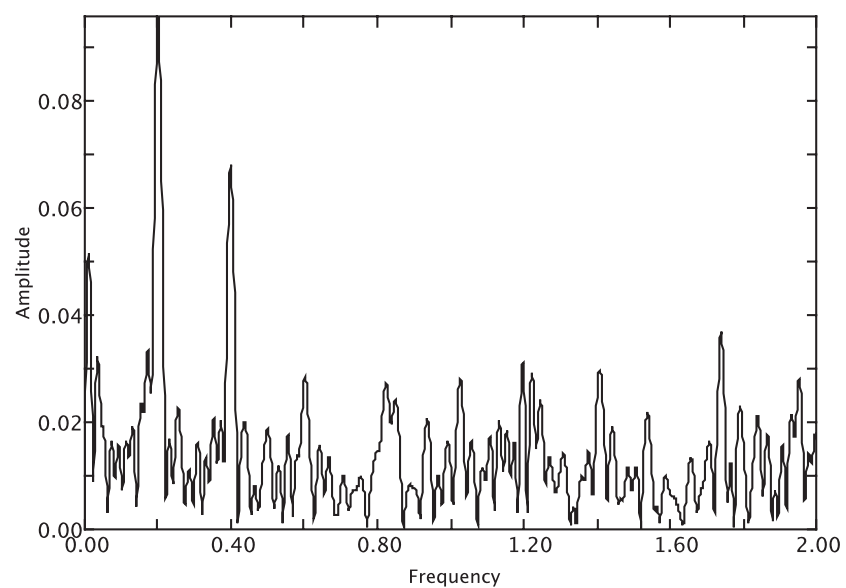

Figure 4. Power spectrum of the declination offsets between $+2.5^{\circ}$ and $+52.5^{\circ}$, showing a clear peak at $0.2 \mathrm{deg}^{-1}$, corresponding to the $5^{\circ}$ spacing of plate centres.

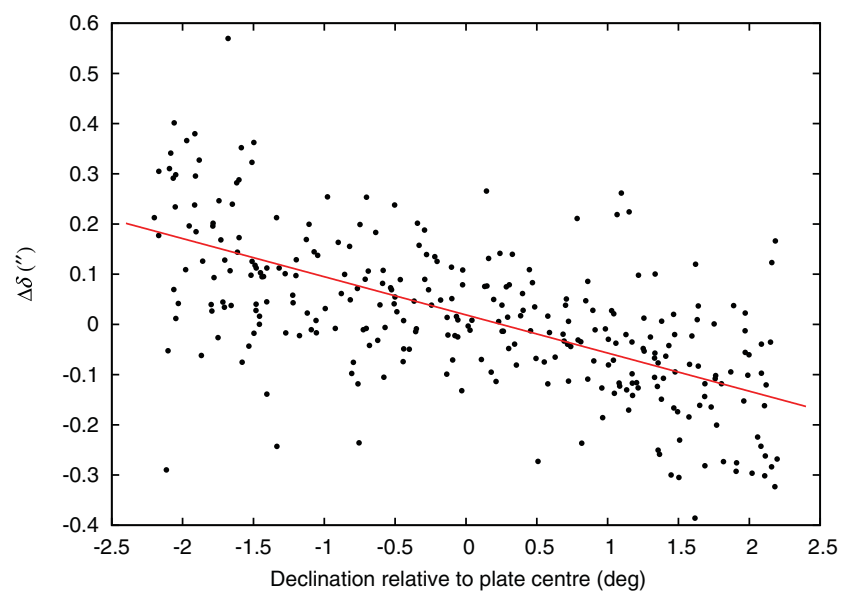

Figure 5. A plot of the declination offset (arcsec, optical minus radio) for sources with declination in the range $+2.5^{\circ}<\delta<+32.5^{\circ}$, co-added on $5^{\circ}$ centres. The red line is the least squares fit to the offsets with slope $-0.076 \pm 0.005$ and intercept $0.019 \pm 0.006$.

in Figure 4. This reveals a clear peak at a frequency of $0.20 \mathrm{deg}^{-1}$ or $5^{\circ}$, the spacing of the plate centres, together with harmonics at 0.4 and $0.6 \mathrm{deg}^{-1}$.

The sawtooth pattern was further confirmed by folding and co-adding the offsets on $5^{\circ}$ centres. Figure 5 shows the declination offsets for sources between declination $+2.5^{\circ}$ and $+32.5^{\circ}$ as a function of distance from the plate centres. Since the plate centres for both northern and southern surveys were defined in B1950 co-ordinates, while the optical and ICRF2 positions are defined in J2000, it was necessary to restrict the plot to $\pm 2.2^{\circ}$ to account for precession in declination between B1950 and J2000.

Nigel Hambly (2013, personal communication) pointed out that the mechanical distortions on POSS-II plates (Hambly et al. 2001c) were significantly larger than on the UK Schmidt plates, and suggested that residual systematics remaining after applying the large (>1 arcsec) corrections could account for the observed pattern in Figure 3, which would naturally have a pitch of $5^{\circ}$. This argument is supported by the absence of a similar pattern in the south.

An alternative (or additional) interpretation of Figures 3 and 5 could be differential refraction, possibly arising from application of the wrong correction for atmospheric refraction in the original SSS reduction. There is tentative evidence that the amplitude of the sawtooth pattern increases towards the northern limit of the POSS-II survey, implying a zenithangle dependence. However, it is difficult to judge whether there is a corresponding dependence north of the Palomar zenith $\left(+33^{\circ}\right)$ because of the falling source density and sky area.

The uniform offset of 0.08 arcsec in the northern RAs suggests a zero-point error. This is surprising since it would have shown up readily in a comparison with ICRF1, as carried out in Hambly et al. (2001c). The mean offsets in the south, with fewer sources, are dominated by the points north of $-20^{\circ}$ and have a smaller overall scatter than in the north.

\section{CONCLUSION}

A detailed comparison has been carried out between optical positions for stellar objects from the SuperCOSMOS J surveys carried out with the UK and Palomar Schmidt telescopes and long-baseline interferometer radio positions that define the ICRF2 reference frame. Subject to selection criteria defined in the paper, we find the overall standard deviations in the position differences to be 0.16 arcsec in both coordinates, with systematic offsets of $<0.1$ arcsec.

In the north, we found a uniform systematic offset of 0.09 arcsec in RA and a sawtooth pattern with mean amplitude $\sim 0.2$ arcsec in declination, with a pitch of $5^{\circ}$ corresponding to the spacing of plate centres. Speculations on the origin of the periodic offsets in declination include residual systematics after correction for the mechanical distortions introduced by the plateholder, and differential errors in correcting for atmospheric refraction.

The overall scatter of the optical positions about ICRF2 is smaller in the south and systematics, while present, are also smaller.

\section{ACKNOWLEDGEMENTS}

We thank Oleg Titov for access to the ICRF2 data, Dennis Stello for the power spectrum in Figure 4, and Nigel Hambly for discussions on the results from this paper.

The POSS-II was made by the California Institute of Technology with funds from the National Science Foundation, the National Aeronautics and Space Administration, the National Geographic Society, the Sloan Foundation, the Samuel Oschin Foundation and the Eastman Kodak Corporation. The Oschin Schmidt telescope is operated by the California Institute of Technology and Palomar Observatory. The UK Schmidt telescope was operated by the Royal Observatory Edinburgh, with funding from the UK Science and Engineering Research Council (later the UK Particle Physics and Astronomy Research Council), until 1988 June, and 
thereafter by the Anglo-Australian Observatory. The blue plates of the southern Sky Atlas and its Equatorial Extension (together known as the SERC-J/EJ) were taken with the UK Schmidt Telescope. All data retrieved from URLs described herein are subject to the copyright given in this copyright summary. Copyright information specific to individual plates is provided in the downloaded FITS headers.

\section{REFERENCES}

Deutsch, E. W. 1999, AJ, 118, 1882

Fey, A., Gordon, G., \& Jacobs, C. (ed.) 2009, The Second Realization of the International Celestial Reference Frame by VLBI, IERS Technical Notes 35, Verlad des Bundesamts fur Kartographie und Geodasie, Frankfurt am Main

Fomalont, E., Johnston, K., Fey, A., Boboltz, D., Oyama, T., \& Honma, M. 2011, AJ, 141, 91

Hambly, N. C., Miller, L., MacGillivray, H. T., Herd, J. T., \& Cormack, W. A. 1998, MNRAS, 298, 897
Hambly, N. C., et al. 2001a, MNRAS, 326, 1279

Hambly, N. C., Irwin, M. J., \& MacGillivray, H. T. 2001b, MNRAS, 326,1295

Hambly, N. C., Davenhall, A. C., Irwin, M. J., \& MacGillivray, H. T. 2001c, MNRAS, 326, 1315

Høg, E., et al. 2000, A\&A, 355, L27

Ma, C., et al. 1998, AJ, 116, 516

Monet, D., et al. 1998, USNO-A2.0: A Catalog of Astrometric Standards (Washington: US Nav. Obs.)

Perryman, M. A. C., et al. 1997, A\&A, 323, L49

Perryman, M. A. C., et al. 2001, A\&A, 369, 339

Mignard, F., \& Klioner, S. 2012, A\&A, 547, A59

Titov, O., Jauncey, D. L., Johnston, H. M., Hunstead, R. W., \& Christensen, L. 2011, AJ, 142, 165

Titov, O., Stanford, L. M., Johnston, H. M., Pursimo, T., Hunstead, R. W., Jauncey, D. L., Maslennikov, K. \& Boldycheva, A. 2013, AJ, 146,10

Urban, S. E., Corbin, T. E., \& Wycoff, G. L. 1998, AJ, 115, 2161 